Supplementary Material for

\title{
Seasonal patterns in Arctic planktonic metabolism \\ (Fram Strait - Svalbard region)
}

Raquel Vaquer-Sunyer*, Carlos M. Duarte, Johnna Holding, Aurore Regaudie-de-Gioux, Lara S. García-Corral, Marit Reigstad and Paul Wassmann

*Corresponding author: raquel@imedea.uib-csic.es

\section{This PDF includes:}

Supplementary Table S1 
Table S1. Planktonic metabolic rates $\left(\mathrm{mmol} \mathrm{O}_{2} \mathrm{~m}^{-3} \mathrm{~d}^{-1}\right)$ for all stations and cruises conducted in the present study. Rates are given as gross primary production (GPP), net community production (NCP) and community respiration (CR) and their associated standard error (SE). Values for the ratio between GPP and CR and between NCP and GPP are also reported.

\begin{tabular}{|c|c|c|c|c|c|c|c|c|c|c|c|c|c|c|}
\hline Cruise & Station & date & depth (m) & $\begin{array}{c}\text { Mixed layer } \\
\text { depth }(\mathrm{m})\end{array}$ & $\begin{array}{c}\mathrm{NCP} \\
(\mathrm{mmol} / \mathrm{m} 3 / \mathrm{d})\end{array}$ & SE & $\begin{array}{c}\mathrm{CR} \\
(\mathrm{mmol} / \mathrm{m} 3 / \mathrm{d})\end{array}$ & SE & $\begin{array}{c}\text { GPP } \\
(\mathrm{mmol} / \mathrm{m} 3 / \mathrm{d})\end{array}$ & $\mathrm{SE}$ & GPP/CR & SE & NCP/GPP & SE \\
\hline ATOS & $1 \mathrm{~b}$ & $01 / 07 / 07$ & 1 & 7.0 & 0.16 & 0.62 & 0.43 & 0.59 & 0.59 & 0.26 & 1.37 & 1.43 & 0.27 & 3.93 \\
\hline ATOS & $1 b$ & $01 / 07 / 07$ & 15 & 7.0 & 0.68 & 0.59 & 0.54 & 0.38 & 1.23 & 0.54 & 2.27 & 0.83 & 0.56 & 0.96 \\
\hline ATOS & $1 \mathrm{~b}$ & $01 / 07 / 07$ & 30 & 7.0 & -3.70 & 1.64 & 3.74 & 1.07 & 0.05 & 1.48 & 0.01 & & -78.95 & 31.59 \\
\hline ATOS & $2 b$ & $02 / 07 / 07$ & 1 & & 1.40 & 0.31 & 0.24 & 0.25 & 1.64 & 0.33 & 6.87 & 1.05 & 0.85 & 0.30 \\
\hline ATOS & $2 b$ & $02 / 07 / 07$ & 10 & & -4.20 & 0.88 & & & & & & & & \\
\hline ATOS & $2 b$ & $02 / 07 / 07$ & 30 & & -1.28 & 0.32 & 2.95 & 0.64 & 1.67 & 0.68 & 0.57 & 0.46 & -0.77 & 0.48 \\
\hline ATOS & $3 b$ & 03/07/07 & 1 & 23.0 & 0.69 & 0.36 & 1.61 & 0.33 & 2.30 & 0.32 & 1.43 & 0.25 & 0.30 & 0.55 \\
\hline ATOS & $3 b$ & 03/07/07 & 10 & 23.0 & 0.09 & 0.28 & 1.98 & 0.59 & 2.08 & 0.58 & 1.05 & 0.41 & 0.05 & 3.01 \\
\hline ATOS & $3 b$ & 03/07/07 & 26 & 23.0 & -1.16 & 0.75 & 2.71 & 0.65 & 1.55 & 0.81 & 0.57 & 0.57 & -0.75 & 0.83 \\
\hline ATOS & $4 \mathrm{~b}$ & $04 / 07 / 07$ & 1 & 9.0 & 0.39 & 0.26 & 1.94 & 0.32 & 2.34 & 0.29 & 1.20 & 0.21 & 0.17 & 0.68 \\
\hline ATOS & $4 \mathrm{~b}$ & $04 / 07 / 07$ & 10 & 9.0 & 1.16 & 0.27 & 1.00 & 0.57 & 2.15 & 0.59 & 2.17 & 0.64 & 0.54 & 0.36 \\
\hline ATOS & $4 b$ & $04 / 07 / 07$ & 32 & 9.0 & -0.77 & 0.81 & 3.11 & 0.36 & 2.34 & 0.84 & 0.75 & 0.38 & -0.33 & 1.11 \\
\hline ATOS & $5 b$ & $05 / 07 / 07$ & 1 & 6.0 & 6.04 & 0.32 & 2.11 & 0.22 & 8.15 & 0.32 & 3.87 & 0.11 & 0.74 & 0.07 \\
\hline ATOS & $5 b$ & $05 / 07 / 07$ & 5 & 6.0 & 10.55 & 0.60 & 3.35 & 0.28 & 13.89 & 0.59 & 4.15 & 0.09 & 0.76 & 0.07 \\
\hline ATOS & $5 b$ & $05 / 07 / 07$ & 15 & 6.0 & 7.23 & 2.55 & 2.78 & 0.46 & 10.01 & 2.54 & 3.60 & 0.30 & 0.72 & 0.44 \\
\hline ATOS & $6 a$ & $06 / 07 / 07$ & 1 & 13.0 & 4.87 & 0.57 & 3.03 & 0.52 & 7.89 & 0.64 & 2.61 & 0.19 & 0.62 & 0.14 \\
\hline ATOS & $6 a$ & $06 / 07 / 07$ & 20 & 13.0 & -4.86 & 0.33 & 11.68 & 0.28 & 6.82 & 0.35 & 0.58 & 0.06 & -0.71 & 0.08 \\
\hline ATOS & $6 a$ & $06 / 07 / 07$ & 36 & 13.0 & 2.15 & 0.74 & 1.57 & 0.31 & 3.72 & 0.68 & 2.37 & 0.27 & 0.58 & 0.39 \\
\hline ATOS & $9 \mathrm{a}$ & $07 / 07 / 07$ & 1 & 5.0 & 12.68 & 0.59 & 1.60 & 1.16 & 14.28 & 1.27 & 8.93 & 0.73 & 0.89 & 0.10 \\
\hline ATOS & $9 a$ & $07 / 07 / 07$ & 5 & 5.0 & 1.93 & 1.48 & 4.58 & 1.21 & 6.51 & 0.90 & 1.42 & 0.30 & 0.30 & 0.78 \\
\hline ATOS & $9 a$ & $07 / 07 / 07$ & 15 & 5.0 & 0.61 & 1.14 & 2.56 & 0.24 & 3.17 & 1.13 & 1.24 & 0.37 & 0.19 & 1.90 \\
\hline ATOS & $12 \mathrm{a}$ & $08 / 07 / 07$ & 1 & 8.0 & 7.06 & 0.34 & 3.42 & 0.33 & 10.48 & 0.25 & 3.06 & 0.10 & 0.67 & 0.05 \\
\hline ATOS & $12 \mathrm{a}$ & $08 / 07 / 07$ & 5 & 8.0 & 8.99 & 1.02 & 3.59 & 0.43 & 12.58 & 1.09 & 3.50 & 0.15 & 0.71 & 0.14 \\
\hline ATOS & $12 \mathrm{a}$ & $08 / 07 / 07$ & 10 & 8.0 & 3.06 & 2.02 & 4.75 & 0.60 & 7.81 & 2.06 & 1.65 & 0.29 & 0.39 & 0.71 \\
\hline ATOS & $15 \mathrm{a}$ & $09 / 07 / 07$ & 1 & 16.0 & 22.71 & 0.66 & 2.53 & 0.23 & 25.24 & 0.66 & 9.99 & 0.09 & 0.90 & 0.04 \\
\hline ATOS & $15 \mathrm{a}$ & $09 / 07 / 07$ & 5 & 16.0 & 20.37 & 0.25 & 4.07 & 0.31 & 24.44 & 0.29 & 6.01 & 0.08 & 0.83 & 0.02 \\
\hline ATOS & $15 \mathrm{a}$ & $09 / 07 / 07$ & 20 & 16.0 & 10.88 & 2.65 & 2.43 & 0.32 & 13.31 & 2.66 & 5.47 & 0.24 & 0.82 & 0.32 \\
\hline ATOS & $18 \mathrm{a}$ & $10 / 07 / 07$ & 1 & 15.0 & 1.97 & 0.52 & 1.86 & 0.53 & 3.82 & 0.29 & 2.06 & 0.29 & 0.51 & 0.28 \\
\hline ATOS & $18 \mathrm{a}$ & $10 / 07 / 07$ & 5 & 15.0 & 3.16 & 0.29 & 1.74 & 0.38 & 4.90 & 0.33 & 2.82 & 0.23 & 0.64 & 0.11 \\
\hline ATOS & $18 \mathrm{a}$ & $10 / 07 / 07$ & 27 & 15.0 & 1.08 & 0.44 & 2.73 & 1.16 & 3.81 & 1.13 & 1.39 & 0.52 & 0.28 & 0.51 \\
\hline
\end{tabular}


Mixed layer

NCP

CR GPP

\begin{tabular}{|c|c|c|c|c|c|c|c|c|c|c|c|c|c|c|}
\hline Cruise & Station & date & depth (m) & depth $(m)$ & $(\mathrm{mmol} / \mathrm{m} 3 / \mathrm{d})$ & SE & $(\mathrm{mmol} / \mathrm{m} 3 / \mathrm{d})$ & SE & $(\mathrm{mmol} / \mathrm{m} 3 / \mathrm{d})$ & SE & $\mathrm{GPP} / \mathrm{CR}$ & SE & NCP/GPP & SE \\
\hline ATOS & $19 a$ & $11 / 07 / 07$ & 2 & 12.0 & 2.82 & 3.72 & 3.18 & 0.69 & 6.00 & 3.77 & 1.89 & 0.67 & 0.47 & 1.46 \\
\hline ATOS & $19 \mathrm{a}$ & 11/07/07 & 12 & 12.0 & 5.94 & 0.26 & 2.80 & 0.33 & 8.74 & 0.35 & 3.12 & 0.13 & 0.68 & 0.06 \\
\hline ATOS & $19 \mathrm{a}$ & $11 / 07 / 07$ & 25 & 12.0 & 0.89 & 0.54 & 1.01 & 0.31 & 1.90 & 0.47 & 1.88 & 0.40 & 0.47 & 0.66 \\
\hline ATOS & $20 \mathrm{a}$ & $12 / 07 / 07$ & 1 & 17.0 & -0.18 & 0.25 & 3.39 & 0.25 & 3.21 & 0.31 & 0.95 & 0.12 & -0.06 & 1.37 \\
\hline ATOS & $20 \mathrm{a}$ & $12 / 07 / 07$ & 10 & 17.0 & 1.00 & 0.70 & 14.26 & 0.78 & 15.26 & 0.85 & 1.07 & 0.08 & 0.07 & 0.70 \\
\hline ATOS & $20 \mathrm{a}$ & $12 / 07 / 07$ & 25 & 17.0 & -5.64 & 0.66 & 11.65 & 1.68 & 6.02 & 1.80 & 0.52 & 0.33 & -0.94 & 0.32 \\
\hline ATOS & $23 a$ & $13 / 07 / 07$ & 1 & 10.0 & -10.23 & 2.50 & 13.00 & 2.71 & 2.78 & 0.24 & 0.21 & 0.22 & -3.68 & 0.26 \\
\hline ATOS & $23 a$ & $13 / 07 / 07$ & 10 & 10.0 & -7.54 & 1.79 & 12.92 & 1.94 & 5.38 & 1.05 & 0.42 & 0.25 & -1.40 & 0.31 \\
\hline ATOS & $23 a$ & $13 / 07 / 07$ & 24 & 10.0 & 3.39 & 0.52 & 3.51 & 1.53 & 6.90 & 1.60 & 1.97 & 0.49 & 0.49 & 0.28 \\
\hline ATOS & $26 \mathrm{a}$ & $14 / 07 / 07$ & 1 & 10.0 & -0.82 & 0.32 & 2.14 & 0.18 & 1.32 & 0.33 & 0.62 & 0.26 & -0.62 & 0.46 \\
\hline ATOS & $26 \mathrm{a}$ & $14 / 07 / 07$ & 15 & 10.0 & -12.94 & 4.14 & 18.52 & 4.15 & 5.58 & 0.60 & 0.30 & 0.25 & -2.32 & 0.34 \\
\hline ATOS & $26 \mathrm{a}$ & $14 / 07 / 07$ & 27 & 10.0 & 4.61 & 1.50 & 3.48 & 0.36 & 8.09 & 1.51 & 2.32 & 0.21 & 0.57 & 0.37 \\
\hline ATOS & $27 \mathrm{a}$ & $15 / 07 / 07$ & 1 & & -4.77 & 8.05 & 20.88 & 1.06 & 16.11 & 7.98 & 0.77 & 0.50 & -0.30 & 1.76 \\
\hline ATOS & $27 \mathrm{a}$ & $15 / 07 / 07$ & 15 & & -21.72 & 1.88 & 29.20 & 1.81 & 7.49 & 0.64 & 0.26 & 0.11 & -2.90 & 0.12 \\
\hline ATOS & $27 \mathrm{a}$ & $15 / 07 / 07$ & 30 & & 6.04 & 0.72 & 1.16 & 0.72 & 7.21 & 0.48 & 6.19 & 0.62 & 0.84 & 0.14 \\
\hline ATOS & $33 a$ & 17/07/07 & 1 & 5.0 & -0.75 & 0.43 & 2.41 & 0.31 & 1.66 & 0.47 & 0.69 & 0.31 & -0.45 & 0.64 \\
\hline ATOS & $33 a$ & $17 / 07 / 07$ & 10 & 5.0 & 4.26 & 0.41 & 3.42 & 0.39 & 7.68 & 0.39 & 2.24 & 0.13 & 0.55 & 0.11 \\
\hline ATOS & $33 a$ & $17 / 07 / 07$ & 22 & 5.0 & -0.27 & 0.63 & 1.21 & 0.56 & 0.93 & 0.49 & 0.77 & 0.70 & -0.29 & 2.36 \\
\hline ATOS & $36 \mathrm{~b}$ & $18 / 07 / 07$ & 1 & 14.0 & 12.67 & 0.36 & & 0.29 & & 0.26 & & & & \\
\hline ATOS & $36 \mathrm{~b}$ & $18 / 07 / 07$ & 14 & 14.0 & 14.33 & 0.49 & & 0.47 & & 0.47 & & & & \\
\hline ATOS & $36 \mathrm{~b}$ & $18 / 07 / 07$ & 30 & 14.0 & 12.37 & 0.44 & & 0.46 & & 0.45 & & & & \\
\hline ATOS & $39 a$ & 19/07/07 & 1 & 8.0 & -1.16 & 1.06 & 1.20 & 0.68 & 0.05 & 0.94 & 0.04 & 20.51 & -25.38 & 20.53 \\
\hline ATOS & $39 a$ & 19/07/07 & 5 & 8.0 & 0.46 & 0.46 & 1.31 & 0.57 & 1.78 & 0.56 & 1.35 & 0.54 & 0.26 & 1.03 \\
\hline ATOS & $39 a$ & $19 / 07 / 07$ & 32 & 8.0 & 1.68 & 0.56 & 2.65 & 2.25 & 4.33 & 2.26 & 1.63 & 0.99 & 0.39 & 0.62 \\
\hline ATOS & $42 a$ & $20 / 07 / 07$ & 1 & & 0.43 & 0.56 & 1.05 & 0.52 & 1.48 & 0.55 & 1.41 & 0.62 & 0.29 & 1.34 \\
\hline ATOS & $42 a$ & $20 / 07 / 07$ & 10 & & 8.94 & 0.83 & 3.16 & 1.60 & 12.11 & 1.77 & 3.83 & 0.53 & 0.74 & 0.17 \\
\hline ATOS & $42 \mathrm{a}$ & $20 / 07 / 07$ & 20 & & 3.60 & 1.57 & 1.79 & 0.59 & 5.39 & 1.60 & 3.02 & 0.44 & 0.67 & 0.53 \\
\hline ATOS & $43 a$ & $22 / 07 / 07$ & 1 & 13 & -7.23 & 0.58 & 12.72 & 0.66 & 5.50 & 0.60 & 0.43 & 0.12 & -1.31 & 0.13 \\
\hline ATOS & $43 a$ & $22 / 07 / 07$ & 10 & 13.0 & -3.84 & 1.39 & 9.40 & 1.23 & 5.56 & 1.51 & 0.59 & 0.30 & -0.69 & 0.45 \\
\hline ATOS & $43 a$ & $22 / 07 / 07$ & 20 & 13.0 & -1.62 & 1.23 & 12.81 & 1.55 & 11.19 & 1.96 & 0.87 & 0.21 & -0.14 & 0.78 \\
\hline ATOS & $46 a$ & $23 / 07 / 07$ & 1 & 16.0 & -9.56 & 0.51 & 10.10 & 0.53 & 0.54 & 0.21 & 0.05 & 0.39 & -17.79 & 0.39 \\
\hline ATOS & $46 a$ & $23 / 07 / 07$ & 14 & 16.0 & -3.45 & 0.47 & 10.22 & 0.37 & 6.76 & 0.39 & 0.66 & 0.07 & -0.51 & 0.15 \\
\hline ATOS & $46 \mathrm{a}$ & $23 / 07 / 07$ & 32 & 16.0 & -8.05 & 0.42 & 8.85 & 0.38 & 0.80 & 0.31 & 0.09 & 0.40 & -10.08 & 0.40 \\
\hline ATOS & $49 a$ & $24 / 07 / 07$ & 1 & & -5.61 & 0.58 & 8.36 & 0.86 & 2.76 & 0.78 & 0.33 & 0.30 & -2.03 & 0.30 \\
\hline
\end{tabular}


NCP

CR GPP

\begin{tabular}{|c|c|c|c|c|c|c|c|c|c|c|c|c|c|c|}
\hline Cruise & Station & date & depth (m) & depth $(\mathrm{m})$ & $(\mathrm{mmol} / \mathrm{m} 3 / \mathrm{d})$ & SE & $(\mathrm{mmol} / \mathrm{m} 3 / \mathrm{d})$ & SE & $(\mathrm{mmol} / \mathrm{m} 3 / \mathrm{d})$ & SE & GPP/CR & SE & NCP/GPP & SE \\
\hline ATOS & $49 a$ & $24 / 07 / 07$ & 10 & & -6.37 & 0.41 & 7.42 & 0.47 & 1.05 & 0.54 & 0.14 & 0.52 & -6.05 & 0.52 \\
\hline ATOS & $49 a$ & $24 / 07 / 07$ & 30 & & -4.67 & 0.79 & 7.52 & 1.31 & 2.85 & 1.45 & 0.38 & 0.54 & -1.64 & 0.53 \\
\hline IAOOS 2007 & $\mathrm{FS} 002 \mathrm{Bb}$ & $16 / 4 / 07$ & 1 & 15.2 & 3.34 & 0.33 & & 0.19 & & 0.27 & & & & \\
\hline IAOOS 2007 & $\mathrm{FS} 002 \mathrm{Bb}$ & $16 / 4 / 07$ & 5 & 15.2 & 2.06 & 0.38 & & 0.52 & & 0.38 & & & & \\
\hline IAOOS 2007 & $\mathrm{FS} 002 \mathrm{Bb}$ & $16 / 4 / 07$ & 10 & 15.2 & 10.96 & 2.49 & & & & & & & & \\
\hline IAOOS 2007 & $\mathrm{FS} 002 \mathrm{Bb}$ & $16 / 4 / 07$ & 20 & 15.2 & -0.58 & 0.48 & 1.06 & 0.48 & 0.48 & 0.41 & 0.45 & 0.97 & -1.21 & 1.19 \\
\hline IAOOS 2007 & $\mathrm{FS} 003 \mathrm{Bb}$ & $18 / 4 / 07$ & 1 & & 0.48 & 8.51 & & 7.61 & & 0.34 & & & & \\
\hline IAOOS 2007 & FS003Bb & $18 / 4 / 07$ & 10 & & -0.02 & 26.71 & & 35.43 & & 1.07 & & & & \\
\hline IAOOS 2007 & $\mathrm{FS} 003 \mathrm{Bb}$ & $18 / 4 / 07$ & 20 & & 0.04 & 19.18 & 1.73 & 19.47 & 1.88 & 0.38 & 1.09 & 11.27 & 0.02 & \\
\hline IAOOS 2007 & $\mathrm{FS} 004 \mathrm{Cb}$ & $22 / 4 / 07$ & 5 & 14 & 0.01 & 0.33 & 0.33 & 0.32 & 0.33 & 0.42 & 1.02 & 1.60 & 0.02 & 63.04 \\
\hline IAOOS 2007 & $\mathrm{FS} 004 \mathrm{Cb}$ & $22 / 4 / 07$ & 10 & 14 & 0.29 & 0.34 & 0.01 & 0.42 & 0.30 & 0.27 & 28.50 & 39.88 & 0.96 & 1.48 \\
\hline IAOOS 2007 & $\mathrm{FS} 004 \mathrm{Cb}$ & $22 / 4 / 07$ & 20 & 14 & 0.09 & 0.42 & & 0.63 & & 0.59 & & & & \\
\hline IAOOS 2007 & $\mathrm{FS} 005 \mathrm{Cb}$ & $25 / 4 / 07$ & 5 & 16 & 1.82 & 0.49 & & 0.47 & & 0.52 & & & & \\
\hline IAOOS 2007 & $\mathrm{FS} 005 \mathrm{Cb}$ & $25 / 4 / 07$ & 10 & 16 & 1.52 & 0.19 & & 0.15 & & 0.20 & & & & \\
\hline IAOOS 2007 & $\mathrm{FS} 005 \mathrm{Cb}$ & $25 / 4 / 07$ & 20 & 16 & 1.88 & 0.22 & & 0.29 & & 0.32 & & & & \\
\hline IAOOS 2008 & FS080013 & $24 / 04 / 08$ & 1 & 22 & 1.98 & 0.16 & & 0.12 & & 0.14 & & & & \\
\hline IAOOS 2008 & FS080013 & $24 / 04 / 08$ & 5 & 22 & 4.25 & 0.20 & & 0.44 & & 0.47 & & & & \\
\hline IAOOS 2008 & FS080013 & $24 / 04 / 08$ & 10 & 22 & 2.34 & 0.53 & & 0.53 & & 0.43 & & & & \\
\hline IAOOS 2008 & FS080013 & $24 / 04 / 08$ & 20 & 22 & -1.11 & 0.53 & & 0.41 & & 0.46 & & & & \\
\hline IAOOS 2008 & FS080032 & $29 / 04 / 08$ & 1 & $>20$ & 2.72 & 0.93 & & 0.74 & & 1.12 & & & & \\
\hline IAOOS 2008 & FS080032 & $29 / 04 / 08$ & 5 & $>20$ & 3.70 & 0.51 & & 0.30 & & 0.45 & & & & \\
\hline IAOOS 2008 & FS080032 & $29 / 04 / 08$ & 10 & $>20$ & 3.25 & 0.38 & & 0.32 & & 0.34 & & & & \\
\hline IAOOS 2008 & FS080032 & $29 / 04 / 08$ & 20 & $>20$ & 8.46 & 0.30 & & 0.21 & & 0.39 & & & & \\
\hline IAOOS 2008 & FS080049 & $05 / 05 / 08$ & 1 & $>19$ & -0.44 & 0.49 & 1.72 & 0.47 & 1.28 & 0.32 & 0.75 & 0.37 & -0.34 & 1.15 \\
\hline IAOOS 2008 & FS080049 & 05/05/08 & 5 & $>19$ & -0.70 & 0.16 & 0.82 & 0.16 & 0.12 & 0.12 & 0.14 & 1.01 & -6.03 & 1.02 \\
\hline IAOOS 2008 & FS080049 & $05 / 05 / 08$ & 10 & $>19$ & -0.54 & 0.18 & & 0.55 & & 0.54 & & & & \\
\hline IAOOS 2008 & FS080049 & $05 / 05 / 08$ & 20 & $>19$ & 0.92 & 1.42 & 1.00 & 0.23 & 1.93 & 1.42 & 1.92 & 0.77 & 0.48 & 1.71 \\
\hline JM-2008 & 321 & $31 / 7 / 08$ & 1 & 30.3 & 0.16 & 0.52 & 1.72 & 0.52 & 1.88 & 0.23 & 1.09 & 0.33 & 0.09 & 3.27 \\
\hline JM-2008 & 321 & $31 / 7 / 08$ & 10 & 30.3 & 0.06 & 0.23 & 2.84 & 0.16 & 2.90 & 0.19 & 1.02 & 0.09 & 0.02 & 3.67 \\
\hline JM-2008 & 321 & $31 / 7 / 08$ & 20 & 30.3 & 0.57 & 0.69 & 0.61 & 0.70 & 1.17 & 0.35 & 1.94 & 1.19 & 0.48 & 1.24 \\
\hline JM-2008 & 321 & $31 / 7 / 08$ & 30 & 30.3 & 0.40 & 0.41 & 2.28 & 0.54 & 2.68 & 0.39 & 1.17 & 0.28 & 0.15 & 1.03 \\
\hline JM-2008 & 324 & $1 / 8 / 08$ & 1 & & 0.54 & 0.32 & 0.80 & 0.31 & 1.35 & 0.20 & 1.67 & 0.41 & 0.40 & 0.61 \\
\hline JM-2008 & 324 & $1 / 8 / 08$ & 5 & & 0.02 & 0.25 & 1.83 & 0.30 & 1.84 & 0.22 & 1.01 & 0.20 & 0.01 & 16.47 \\
\hline JM-2008 & 324 & $1 / 8 / 08$ & 10 & & 0.67 & 0.26 & 2.40 & 0.25 & 3.07 & 0.15 & 1.28 & 0.11 & 0.22 & 0.40 \\
\hline
\end{tabular}


Mixed layer

NCP

CR GPP

\begin{tabular}{|c|c|c|c|c|c|c|c|c|c|c|c|c|c|c|}
\hline Cruise & Station & date & depth (m) & depth (m) & $(\mathrm{mmol} / \mathrm{m} 3 / \mathrm{d})$ & $\mathrm{SE}$ & $(\mathrm{mmol} / \mathrm{m} 3 / \mathrm{d})$ & $\mathrm{SE}$ & $(\mathrm{mmol} / \mathrm{m} 3 / \mathrm{d})$ & SE & GPP/CR & SE & NCP/GPP & SE \\
\hline JM-2008 & 324 & $1 / 8 / 08$ & 20 & & 1.30 & 0.36 & 3.22 & 0.87 & 4.52 & 0.83 & 1.40 & 0.33 & 0.29 & 0.33 \\
\hline JM-2008 & 326 & $2 / 8 / 08$ & 1 & & 0.16 & 0.52 & 1.72 & 0.52 & 1.88 & 0.23 & 1.09 & 0.33 & 0.09 & 3.27 \\
\hline JM-2008 & 326 & $2 / 8 / 08$ & 10 & & 0.06 & 0.23 & 2.84 & 0.16 & 2.90 & 0.19 & 1.02 & 0.09 & 0.02 & 3.67 \\
\hline JM-2008 & 326 & $2 / 8 / 08$ & 20 & & 0.40 & 0.41 & 2.28 & 0.54 & 2.68 & 0.39 & 1.17 & 0.28 & 0.15 & 1.03 \\
\hline JM-2008 & 326 & $2 / 8 / 08$ & 25 & & 0.57 & 0.69 & 0.61 & 0.70 & 1.17 & 0.35 & 1.94 & 1.19 & 0.48 & 1.24 \\
\hline JM-2008 & 327 & $3 / 8 / 08$ & 1 & & 0.26 & 0.43 & 0.43 & 0.41 & 0.69 & 0.15 & 1.62 & 0.99 & 0.38 & 1.67 \\
\hline JM-2008 & 327 & $3 / 8 / 08$ & 5 & & -0.04 & 0.19 & 1.01 & 1.01 & 0.97 & 1.00 & 0.96 & 1.43 & -0.04 & 5.09 \\
\hline JM-2008 & 327 & $3 / 8 / 08$ & 10 & & -1.55 & 0.59 & 2.92 & 0.54 & 1.36 & 0.43 & 0.47 & 0.37 & -1.14 & 0.49 \\
\hline JM-2008 & 327 & $3 / 8 / 08$ & 20 & & 1.75 & 0.13 & 0.17 & 0.20 & 1.91 & 0.24 & 11.42 & 1.22 & 0.91 & 0.15 \\
\hline JM-2008 & 329 & $4 / 8 / 08$ & 1 & & -0.62 & 0.32 & 0.59 & 0.38 & & 0.36 & & & & \\
\hline JM-2008 & 329 & $4 / 8 / 08$ & 10 & & 1.15 & 0.59 & 2.22 & 0.71 & 3.36 & 0.88 & 1.52 & 0.42 & 0.34 & 0.58 \\
\hline JM-2008 & 329 & $4 / 8 / 08$ & 20 & & 0.71 & 0.38 & 2.72 & 0.34 & 3.43 & 0.19 & 1.26 & 0.14 & 0.21 & 0.54 \\
\hline JM-2008 & 329 & $4 / 8 / 08$ & 25 & & 0.12 & 0.17 & 1.00 & 0.12 & 1.12 & 0.14 & 1.12 & 0.17 & 0.11 & 1.45 \\
\hline JM-2008 & 330 & $5 / 8 / 08$ & 1 & & -0.43 & 0.29 & 0.67 & 0.23 & 0.24 & 0.22 & 0.36 & 0.97 & -1.81 & 1.13 \\
\hline JM-2008 & 330 & $5 / 8 / 08$ & 5 & & 0.13 & 0.31 & & 0.93 & & 0.77 & & & & \\
\hline JM-2008 & 330 & $5 / 8 / 08$ & 10 & & -0.69 & 0.39 & 1.99 & 0.49 & 1.31 & 0.30 & 0.66 & 0.33 & -0.53 & 0.61 \\
\hline JM-2008 & 330 & $5 / 8 / 08$ & 20 & & -1.27 & 0.97 & 1.75 & 1.03 & 0.48 & 0.45 & 0.28 & & -2.63 & 1.20 \\
\hline ARCTOS & 419 & $29 / 11 / 06$ & 1 & 67.7 & -0.02 & 1.25 & 0.02 & 0.88 & 0.00 & 0.88 & 0.00 & & & \\
\hline ARCTOS & 426 & $30 / 11 / 06$ & 1 & & -0.37 & 1.03 & 0.37 & 0.73 & 0.00 & 0.73 & 0.00 & & & \\
\hline ARCTOS & $\mathrm{P} 1$ & $1 / 12 / 06$ & 1 & & -0.19 & 1.16 & 0.19 & 0.82 & 0.00 & 0.82 & 0.00 & & & \\
\hline ARCTOS & $\mathrm{Kb} 3$ & $2 / 12 / 06$ & 1 & & -0.69 & 1.78 & 0.69 & 1.26 & 0.00 & 1.26 & 0.00 & & & \\
\hline ARCTOS & $\mathrm{Kb} 5$ & $2 / 12 / 06$ & 1 & & -1.49 & 0.82 & 1.49 & 0.58 & 0.00 & 0.58 & 0.00 & & & \\
\hline ARCTOS & $\mathrm{Kb} 1$ & $2 / 12 / 06$ & 1 & & -2.56 & 0.70 & 2.56 & 0.50 & 0.00 & 0.50 & 0.00 & & & \\
\hline ARCTOS & $\mathrm{Kb} 4$ & $3 / 12 / 06$ & 1 & & -0.57 & 0.64 & 0.57 & 0.45 & 0.00 & 0.45 & 0.00 & & & \\
\hline АTP 09 & 1 & $17 / 6 / 2009$ & 2 & $>51.5$ & 4.30 & 2.47 & 3.57 & 1.60 & 7.87 & 1.59 & 2.20 & 0.49 & 0.55 & 0.61 \\
\hline ATP 09 & 1 & $17 / 6 / 2009$ & 25 & $>51.5$ & 13.07 & 1.09 & 4.66 & 0.58 & 17.73 & 0.55 & 3.80 & 0.13 & 0.74 & 0.09 \\
\hline АТР 09 & 1 & $17 / 6 / 2009$ & 50 & $>51.5$ & 4.73 & 0.94 & 9.89 & 2.13 & 14.63 & 1.60 & 1.48 & 0.24 & 0.32 & 0.23 \\
\hline ATP 09 & 2 & $18 / 6 / 2009$ & 1 & 16.8 & 1.38 & 0.37 & & 0.93 & & 0.90 & & & & \\
\hline ATP 09 & 2 & $18 / 6 / 2009$ & 17 & 16.8 & -1.53 & 2.48 & 4.63 & 0.86 & 3.11 & 0.95 & 0.67 & 0.36 & -0.49 & 1.65 \\
\hline АTP 09 & 2 & $18 / 6 / 2009$ & 35 & 16.8 & 62.49 & 1.49 & 1.91 & 0.53 & 64.40 & 1.55 & 33.64 & 0.28 & 0.97 & 0.03 \\
\hline АTP 09 & 3 & $19 / 6 / 2009$ & 1 & 26.7 & 5.20 & 1.01 & 1.68 & 0.47 & 6.87 & 1.06 & 4.10 & 0.32 & 0.76 & 0.25 \\
\hline АТР 09 & 3 & $19 / 6 / 2009$ & 10 & 26.7 & 15.88 & 0.68 & 0.91 & 1.03 & 16.79 & 1.03 & 18.44 & 1.14 & 0.95 & 0.08 \\
\hline ATP 09 & 3 & $19 / 6 / 2009$ & 25 & 26.7 & 15.41 & 1.08 & 3.11 & 0.74 & 18.52 & 1.00 & 5.95 & 0.25 & 0.83 & 0.09 \\
\hline АTP 09 & 4 & $20 / 6 / 2009$ & 1 & 11.9 & -0.05 & 0.40 & 0.82 & 0.75 & 0.77 & 0.69 & 0.94 & 1.28 & -0.06 & 8.83 \\
\hline
\end{tabular}


Mixed layer NCP CR GPP

\begin{tabular}{|c|c|c|c|c|c|c|c|c|c|c|c|c|c|c|}
\hline Cruise & Station & date & depth (m) & depth (m) & $(\mathrm{mmol} / \mathrm{m} 3 / \mathrm{d})$ & $\mathrm{SE}$ & $(\mathrm{mmol} / \mathrm{m} 3 / \mathrm{d})$ & SE & $(\mathrm{mmol} / \mathrm{m} 3 / \mathrm{d})$ & SE & GPP/CR & SE & NCP/GPP & SE \\
\hline ATP 09 & 4 & $20 / 6 / 2009$ & 15 & 11.9 & -1.91 & 0.88 & 0.11 & 1.11 & & 1.06 & & & & \\
\hline ATP 09 & 4 & $20 / 6 / 2009$ & 35 & 11.9 & 2.07 & 0.62 & 1.85 & 0.38 & 3.92 & 0.70 & 2.12 & 0.27 & 0.53 & 0.35 \\
\hline ATP 09 & 5 & $22 / 6 / 2009$ & 1 & & 1.01 & 0.54 & 1.97 & 0.40 & 2.98 & 0.52 & 1.51 & 0.27 & 0.34 & 0.57 \\
\hline ATP 09 & 5 & $22 / 6 / 2009$ & 15 & & 15.08 & 0.69 & 5.06 & 1.11 & 20.14 & 1.11 & 3.98 & 0.23 & 0.75 & 0.07 \\
\hline ATP 09 & 5 & $22 / 6 / 2009$ & 30 & & 12.29 & 1.07 & 2.47 & 0.72 & 14.76 & 1.27 & 5.98 & 0.30 & 0.83 & 0.12 \\
\hline ATP 09 & 6 & $23 / 6 / 2009$ & 1 & & 4.14 & 1.03 & 5.96 & 0.97 & 10.09 & 0.92 & 1.69 & 0.19 & 0.41 & 0.27 \\
\hline ATP 09 & 6 & $23 / 6 / 2009$ & 10 & & 8.08 & 0.63 & 2.29 & 0.72 & 10.37 & 0.72 & 4.52 & 0.32 & 0.78 & 0.10 \\
\hline ATP 09 & 6 & $23 / 6 / 2009$ & 25 & & 16.88 & 1.12 & 0.99 & 0.69 & 17.87 & 0.96 & 18.00 & 0.69 & 0.94 & 0.09 \\
\hline ATP 09 & K4 & $24 / 6 / 2009$ & 1 & 6.9 & 1.96 & 0.75 & 4.73 & 2.07 & 6.69 & 1.86 & 1.41 & 0.52 & 0.29 & 0.47 \\
\hline ATP 09 & K4 & $24 / 6 / 2009$ & 25 & 6.9 & -0.21 & 0.68 & 0.80 & 0.72 & 0.59 & 0.72 & 0.74 & 1.51 & -0.35 & 3.50 \\
\hline ATP 09 & K4 & $24 / 6 / 2009$ & 55 & 6.9 & 0.53 & 0.89 & 5.04 & 0.97 & 5.57 & 1.17 & 1.11 & 0.29 & 0.10 & 1.69 \\
\hline ATP 09 & T16 & $25 / 6 / 09$ & 1 & 12.9 & 6.50 & 1.17 & & 0.59 & & 1.24 & & & & \\
\hline ATP 09 & $\mathrm{~T} 16$ & $25 / 6 / 09$ & 10 & 12.9 & 12.35 & 0.57 & 1.87 & 0.68 & 14.22 & 0.68 & 7.60 & 0.36 & 0.87 & 0.07 \\
\hline ATP 09 & $\mathrm{~T} 16$ & $25 / 6 / 09$ & 25 & 12.9 & 7.59 & 1.18 & & 0.92 & & 0.82 & & & & \\
\hline ATP 2010 & 1 & $4 / 5 / 10$ & 1 & 25.7 & 16.09 & 1.62 & 23.02 & 7.95 & 39.11 & 8.07 & 1.70 & 0.40 & 0.41 & 0.23 \\
\hline ATP 2010 & 1 & $4 / 5 / 10$ & 8 & 25.7 & 18.66 & 1.12 & & & & & & & & \\
\hline ATP 2010 & 1 & $4 / 5 / 10$ & 15 & 25.7 & 16.26 & 0.85 & 3.00 & 1.28 & 19.26 & 1.19 & 6.41 & 0.43 & 0.84 & 0.08 \\
\hline ATP 2010 & 2 & $5 / 5 / 10$ & 1 & 21.8 & 16.29 & 0.65 & & & & & & & & \\
\hline ATP 2010 & 2 & $5 / 5 / 10$ & 5 & 21.8 & 14.44 & 0.70 & 0.83 & 0.86 & 15.28 & 0.78 & 18.34 & 1.03 & 0.95 & 0.07 \\
\hline ATP 2010 & 2 & $5 / 5 / 10$ & 10 & 21.8 & 15.22 & 0.59 & 0.64 & 0.26 & 15.86 & 0.64 & 24.69 & 0.40 & 0.96 & 0.06 \\
\hline ATP 2010 & 3 & $06 / 05 / 10$ & 1 & $>26$ & 2.85 & 1.01 & 0.76 & 0.99 & 3.62 & 0.34 & 4.73 & 1.29 & 0.79 & 0.37 \\
\hline ATP 2010 & 3 & $06 / 05 / 10$ & 8 & $>26$ & 3.86 & 0.53 & 0.81 & 0.60 & 4.67 & 0.60 & 5.80 & 0.75 & 0.83 & 0.19 \\
\hline ATP 2010 & 3 & $06 / 05 / 10$ & 12 & $>26$ & 2.38 & 0.65 & 1.53 & 0.73 & 3.90 & 0.49 & 2.56 & 0.50 & 0.61 & 0.30 \\
\hline ATP 2010 & 3 & $06 / 05 / 10$ & 15 & $>26$ & 1.37 & 0.38 & 0.16 & 0.18 & 1.52 & 0.41 & 9.80 & 1.17 & 0.90 & 0.39 \\
\hline ATP 2010 & 4 & $07 / 05 / 10$ & 1 & $>15$ & 38.76 & 1.63 & 0.41 & 0.73 & 39.17 & 1.85 & 96.67 & 1.80 & 0.99 & 0.06 \\
\hline ATP 2010 & 4 & $07 / 05 / 10$ & 8 & $>15$ & 37.09 & 1.11 & 0.87 & 1.14 & 37.96 & 1.14 & 43.53 & 1.31 & 0.98 & 0.04 \\
\hline ATP 2010 & 4 & $07 / 05 / 10$ & 12 & $>15$ & 34.63 & 0.88 & 2.21 & 0.98 & 36.84 & 1.31 & 16.69 & 0.45 & 0.94 & 0.04 \\
\hline ATP 2010 & 4 & $07 / 05 / 10$ & 15 & $>15$ & 15.16 & 0.90 & 0.74 & 0.36 & 15.90 & 0.90 & 21.48 & 0.49 & 0.95 & 0.08 \\
\hline ATP 2010 & 5 & $08 / 05 / 10$ & 1 & $>15$ & 17.30 & 0.53 & 5.87 & 3.02 & 23.18 & 2.91 & 3.95 & 0.53 & 0.75 & 0.13 \\
\hline ATP 2010 & 5 & $08 / 05 / 10$ & 8 & $>15$ & 14.86 & 1.02 & 1.82 & 1.09 & 16.68 & 1.09 & 9.17 & 0.60 & 0.89 & 0.09 \\
\hline ATP 2010 & 5 & $08 / 05 / 10$ & 15 & $>15$ & 15.16 & 0.90 & 0.74 & 0.36 & 15.90 & 0.90 & 21.48 & 0.49 & 0.95 & 0.08 \\
\hline ATP 2010 & 6 & $09 / 05 / 10$ & 1 & 13.9 & 46.88 & 2.10 & 2.01 & 0.23 & 48.89 & 2.30 & 24.32 & 0.13 & 0.96 & 0.06 \\
\hline ATP 2010 & 6 & $09 / 05 / 10$ & 8 & 13.9 & 34.68 & 1.36 & 1.57 & 1.40 & 36.25 & 1.40 & 23.16 & 0.89 & 0.96 & 0.05 \\
\hline ATP 2010 & 6 & $09 / 05 / 10$ & 12 & 13.9 & 39.14 & 0.65 & 0.71 & 0.42 & 39.84 & 0.64 & 56.31 & 0.59 & 0.98 & 0.02 \\
\hline
\end{tabular}


Mixed layer NCP CR GPP

\begin{tabular}{|c|c|c|c|c|c|c|c|c|c|c|c|c|c|c|}
\hline Cruise & Station & date & depth (m) & depth $(\mathrm{m})$ & $(\mathrm{mmol} / \mathrm{m} 3 / \mathrm{d})$ & $\mathrm{SE}$ & $(\mathrm{mmol} / \mathrm{m} 3 / \mathrm{d})$ & SE & $(\mathrm{mmol} / \mathrm{m} 3 / \mathrm{d})$ & SE & GPP/CR & SE & NCP/GPP & SE \\
\hline ATP 2010 & 6 & $09 / 05 / 10$ & 15 & 13.9 & 37.92 & 1.44 & 0.07 & 0.96 & 37.99 & 1.16 & 549.75 & 13.93 & 1.00 & 0.05 \\
\hline ATP 2010 & 7 & $10 / 05 / 10$ & 1 & & 39.81 & 0.35 & 3.15 & 0.79 & 42.96 & 0.91 & 13.64 & 0.25 & 0.93 & 0.02 \\
\hline ATP 2010 & 7 & $10 / 05 / 10$ & 10 & & 45.95 & 1.04 & 0.54 & 1.01 & 46.49 & 1.06 & 85.87 & 1.87 & 0.99 & 0.03 \\
\hline ATP 2010 & 7 & $10 / 05 / 10$ & 15 & & 47.61 & 0.96 & & & & & & & & \\
\hline ATP 2011 & 215 & $23 / 5 / 11$ & 3 & & 4.35 & 0.50 & & 0.76 & & 0.81 & & & & \\
\hline ATP 2011 & 215 & $23 / 5 / 11$ & 16 & & 11.02 & 0.99 & & 1.52 & & 1.61 & & & & \\
\hline ATP 2011 & 215 & $23 / 5 / 11$ & 22 & & & 0.35 & & 0.27 & 8.56 & 0.39 & & & & \\
\hline ATP 2011 & 217 & $24 / 5 / 11$ & 4 & 13.9 & 32.86 & 0.88 & & 0.38 & & 0.97 & & & & \\
\hline ATP 2011 & 217 & $24 / 5 / 11$ & 9 & 13.9 & 26.59 & 0.87 & 2.45 & 0.42 & 29.04 & 0.91 & 11.85 & 0.17 & 0.92 & 0.05 \\
\hline ATP 2011 & 217 & $24 / 5 / 11$ & 16 & 13.9 & 34.93 & 2.55 & 18.96 & 4.34 & 53.89 & 4.96 & 2.84 & 0.25 & 0.65 & 0.12 \\
\hline ATP 2011 & 219 & $25 / 5 / 11$ & 1 & & 22.63 & 1.61 & & 1.65 & & 0.70 & & & & \\
\hline ATP 2011 & 219 & $25 / 5 / 11$ & 9 & & & 1.56 & & 1.46 & 18.77 & 0.84 & & & & \\
\hline ATP 2011 & 219 & $25 / 5 / 11$ & 15 & & 21.46 & 2.08 & 12.51 & 1.77 & 33.97 & 1.26 & 2.72 & 0.15 & 0.63 & 0.10 \\
\hline ATP 2011 & 223 & $26 / 5 / 11$ & 3 & & 27.04 & 0.83 & 2.91 & 0.28 & 29.95 & 0.78 & 10.31 & 0.10 & 0.90 & 0.04 \\
\hline ATP 2011 & 223 & $26 / 5 / 11$ & 10 & & & 1.31 & & 1.36 & 39.43 & 0.48 & & & & \\
\hline ATP 2011 & 223 & $26 / 5 / 11$ & 18 & & 62.13 & 1.37 & 6.36 & 0.71 & 68.49 & 1.23 & 10.77 & 0.11 & 0.91 & 0.03 \\
\hline ATP 2011 & 226 & $27 / 5 / 11$ & 3 & 41.6 & 2.87 & 0.92 & 3.61 & 0.91 & 6.48 & 0.37 & 1.79 & 0.26 & 0.44 & 0.33 \\
\hline ATP 2011 & 226 & $27 / 5 / 11$ & 10 & 41.6 & 6.67 & 0.45 & 1.17 & 0.62 & 7.84 & 0.62 & 6.70 & 0.53 & 0.85 & 0.10 \\
\hline ATP 2011 & 226 & $27 / 5 / 11$ & 21 & 41.6 & -4.47 & 1.64 & 10.60 & 1.40 & 6.13 & 0.68 & 0.58 & 0.17 & -0.73 & 0.38 \\
\hline ATP 2011 & 229 & $28 / 5 / 11$ & 3 & & 13.34 & 0.48 & 3.11 & 0.51 & 16.45 & 0.43 & 5.29 & 0.17 & 0.81 & 0.04 \\
\hline ATP 2011 & 229 & $28 / 5 / 11$ & 10 & & -8.67 & 0.56 & 15.28 & 0.48 & 6.61 & 0.32 & 0.43 & 0.06 & -1.31 & 0.08 \\
\hline ATP 2011 & 229 & $28 / 5 / 11$ & 16 & & 2.77 & 1.10 & 4.28 & 0.93 & 7.06 & 1.00 & 1.65 & 0.26 & 0.39 & 0.42 \\
\hline ATP 2011 & 232 & $29 / 5 / 11$ & 3 & & & 0.67 & & 0.54 & 13.79 & 0.65 & & & & \\
\hline ATP 2011 & 232 & $29 / 5 / 11$ & 9 & & -13.28 & 0.94 & 40.91 & 0.61 & 27.63 & 0.95 & 0.68 & 0.04 & -0.48 & 0.08 \\
\hline ATP 2011 & 232 & $29 / 5 / 11$ & 20 & & 81.64 & 0.66 & & 0.50 & & 0.70 & & & & \\
\hline ATP 2011 & 234 & $30 / 5 / 11$ & 3 & 22.8 & 38.48 & 1.35 & 4.61 & 0.61 & 43.08 & 1.39 & 9.36 & 0.14 & 0.89 & 0.05 \\
\hline ATP 2011 & 234 & $30 / 5 / 11$ & 8 & 22.8 & & 1.02 & & 0.56 & 54.85 & 0.88 & & & & \\
\hline ATP 2011 & 234 & $30 / 5 / 11$ & 15 & 22.8 & 71.97 & 1.94 & 8.06 & 0.71 & 80.02 & 1.70 & 9.93 & 0.09 & 0.90 & 0.03 \\
\hline ATP 2011 & 240 & $31 / 5 / 11$ & 4 & 10.9 & 1.97 & 0.45 & 1.30 & 0.47 & 3.27 & 0.18 & 2.51 & 0.36 & 0.60 & 0.23 \\
\hline ATP 2011 & 240 & $31 / 5 / 11$ & 15 & 10.9 & 6.90 & 0.43 & 0.41 & 0.30 & 7.31 & 0.41 & 17.80 & 0.72 & 0.94 & 0.08 \\
\hline ATP 2011 & 240 & $31 / 5 / 11$ & 33 & 10.9 & 9.44 & 0.81 & 4.93 & 0.86 & 14.37 & 0.56 & 2.92 & 0.18 & 0.66 & 0.09 \\
\hline ATP 2011 & 243 & $1 / 6 / 11$ & 3 & 30.7 & 8.39 & 0.29 & 3.33 & 0.20 & 11.72 & 0.23 & 3.52 & 0.06 & 0.72 & 0.04 \\
\hline ATP 2011 & 243 & $1 / 6 / 11$ & 12 & 30.7 & 9.09 & 0.49 & 2.88 & 0.31 & 11.97 & 0.55 & 4.15 & 0.12 & 0.76 & 0.07 \\
\hline ATP 2011 & 243 & $1 / 6 / 11$ & 27 & 30.7 & 8.75 & 0.44 & 11.88 & 0.64 & 20.63 & 0.45 & 1.74 & 0.06 & 0.42 & 0.05 \\
\hline
\end{tabular}




\begin{tabular}{|c|c|c|c|c|c|c|c|c|c|c|c|c|c|c|}
\hline Cruise & Station & date & depth (m) & $\begin{array}{c}\text { Mixed layer } \\
\text { depth }(\mathrm{m})\end{array}$ & $\begin{array}{c}\mathrm{NCP} \\
(\mathrm{mmol} / \mathrm{m} 3 / \mathrm{d})\end{array}$ & SE & $\begin{array}{c}\mathrm{CR} \\
(\mathrm{mmol} / \mathrm{m} 3 / \mathrm{d})\end{array}$ & SE & $\begin{array}{c}\text { GPP } \\
(\mathrm{mmol} / \mathrm{m} 3 / \mathrm{d})\end{array}$ & SE & GPP/CR & SE & NCP/GPP & SE \\
\hline ATP 2011 & 246 & $2 / 6 / 11$ & 3 & & 21.46 & 0.47 & 2.24 & 0.37 & 23.70 & 0.40 & 10.57 & 0.17 & 0.91 & 0.03 \\
\hline ATP 2011 & 246 & $2 / 6 / 11$ & 7 & & 24.04 & 0.91 & 2.46 & 0.89 & 26.51 & 0.24 & 10.76 & 0.36 & 0.91 & 0.04 \\
\hline ATP 2011 & 246 & $2 / 6 / 11$ & 13 & & 51.12 & 0.58 & 5.94 & 0.44 & 57.06 & 0.62 & 9.61 & 0.07 & 0.90 & 0.02 \\
\hline ATP 2011 & 249 & $3 / 6 / 11$ & 3 & & 2.02 & 0.25 & 4.64 & 0.67 & 6.67 & 0.63 & 1.44 & 0.17 & 0.30 & 0.16 \\
\hline ATP 2011 & 249 & $3 / 6 / 11$ & 10 & & 4.60 & 0.57 & 2.80 & 0.78 & 7.40 & 0.83 & 2.64 & 0.30 & 0.62 & 0.17 \\
\hline ATP 2011 & 249 & $3 / 6 / 11$ & 17 & & 8.42 & 0.56 & 10.60 & 0.47 & 19.02 & 0.37 & 1.79 & 0.05 & 0.44 & 0.07 \\
\hline
\end{tabular}

